\title{
Application of adaptive models for the determination of the thermal behaviour of a photovoltaic panel
}

\author{
Valerio Lo Brano ${ }^{1}$, Giuseppina Ciulla ${ }^{1}$, Marco Beccali ${ }^{1}$ \\ ${ }^{1}$ DEIM, Dipartimento di Energia, Ingegneria dell'informazione e Modelli matematici, Universi- \\ tà di Palermo, viale delle scienze, Ed.9 90128, Palermo, Italy \\ \{lobrano, ciullaina, marco.beccali\}@dream.unipa.it
}

\begin{abstract}
The use of reliable forecasting models for the PV temperature is necessary for a more correct evaluation of energy and economic performances. Climatic conditions certainly have a remarkable influence on thermo-electric behaviour of the PV panel but the physical system is too complex for an analytical representation. A neural-network-based approach for solar panel temperature modelling is here presented. The models were trained using a set of data collected from a test facility. Simulation results of the trained neural networks are presented and compared with those obtained with an empirical correlation.
\end{abstract}

Keywords: Artificial Neural Network, photovoltaic, cell temperature

\section{Introduction}

Renewable Energy Sources (RES) are important for promoting the competitiveness of the economy of countries, the security of energy supply systems and to improve the environmental protection [1,2]. Generally, RES are easily accessible, inexhaustible and compatible with the environment. Among RES, solar energy has the greatest energy potential and photovoltaic (PV) arrays permit to produce electric power directly from sunlight with no fossil-fuel consumption, no noise, and posing no health and environmental hazards during the operational phase of life. This fact, together with the slow but ongoing decline of conventional energy sources, implies a promising role for PV power-generation systems in the near future. Despite the technological and environmental benefits granted by this technology, the development of PV panels is hindered by economic factors. The high cost of production and installation makes the $\mathrm{PV}$ technology feasible only when public funding is available $[2,3]$.

Furthermore, it is clear that the availability of reliable predictive tools is very important for the dissemination of all renewable energy technologies [4, 5]. In details, from the point of view of the designer and end-users of PV systems, the availability of reliable software tools is essential to optimize the performance of PV systems in the planning phase and finally to correctly assess the economic gain [6]. In order to evaluate the real performance of $\mathrm{PV}$ panels the correct prediction of operating temperature is very important [7]; an increase of few degrees can considerably reduce the conversion efficiency of the system thus reducing the power output.

A reliable tool for predicting the temperature of PV systems is also particularly important in those hybrid systems called PV-Thermal (PVT), which allow the recov-

adfa, p. 1, 2011.

(C) Springer-Verlag Berlin Heidelberg 2011 
ery of thermal energy that otherwise, would be wasted into the surrounding environment [8-10].

The aim of this work is to explore the possibility to offer an alternative method, respect to empirical correlations, which allows modelling the operating temperature of PV devices by using techniques based upon adaptive systems. Adaptive systems, such as Artificial Neural Networks (ANN) should allow to predict, with a fast and reliable procedure, the temperature of the PV module as weather conditions change.

\section{Introduction to Artificial Neural Networks and classification problems}

ANNs emulate some of the functions and capabilities of the human brain and their use is now widespread in the scientific literature, in particular in those physical models where, although the interconnections among some variables are widely demonstrated, the corresponding mathematical functions are not known or they are extremely complex [11-13]. One of the most important area of ANNs is the pattern recognition and the main task of this approach is the classification [14]. What is a classifier? A classifier is an expert system that builds a relation between a variable space A and a vector of labels B. A classifier is able to assign a label to a sample extracted from the space A. Nowadays, many expert systems are used as classifiers, e.g. in order to assign or not the label of "SPAM" to an email sent to our mailbox [15]. The classifier examines some features of the email such as the sender, the object, the text and other variables and then it assigns or not the label of SPAM. Another example of a classifier is an expert system able to recognize people from face images [16] or from the sound of voices [17].

Classification is different from clustering: in a cluster analysis, data are automatically separated in groups characterized by some similarities, called clusters. So, clustering is an unsupervised process that groups the data autonomously. Classification is a supervised process where a user decides the set of labels.

A Neural Network Classifier (NNC) simply judges the distance between a pattern of input variables and some given labels. There are two ways to measure this distance: by numerical methods and non-numerical ones. The numerical techniques measure the above-mentioned distances in deterministic or statistical way. The non-numerical techniques are linked to symbolic processes like fuzzy sets. Making these activities automatic permits to reach the target in a faster and more reliable way. Furthermore, the use of NNCs often makes it possible to identify correlations between data which are so complex that they would be hardly recognized even by an expert human operator.

In time series forecasting problems one of the approaches often used by researchers is represented by ANN based techniques that can be used as an alternative method in the analysis of complex and/or ill-defined engineering problems. ANNs do not require the formulation of a mathematical relation describing a complex natural and/or physical system and have the capability of detecting its hidden structure. Accurate forecasting of time series can be useful in many practical situations and the knowledge of 
variation in the operating temperature of PV can play a remarkable role in the assessment of power output.

\section{The operative temperature of a PV panel}

To design and assess the performances of a PV system, an accurate PV model should predict reliable Current-Voltage $(I-V)$ and Power-Voltage $(P-V)$ curves under real operating conditions [18].

The "five-parameters model" represents the most common equivalent circuit that better describes the electrical behavior of a PV system. The equivalent circuit is composed of a photocurrent source $I_{L}$, a diode in parallel with a shunt resistance $R_{s h}$, and a series resistance $R_{s}$ as shown in Figure 1.

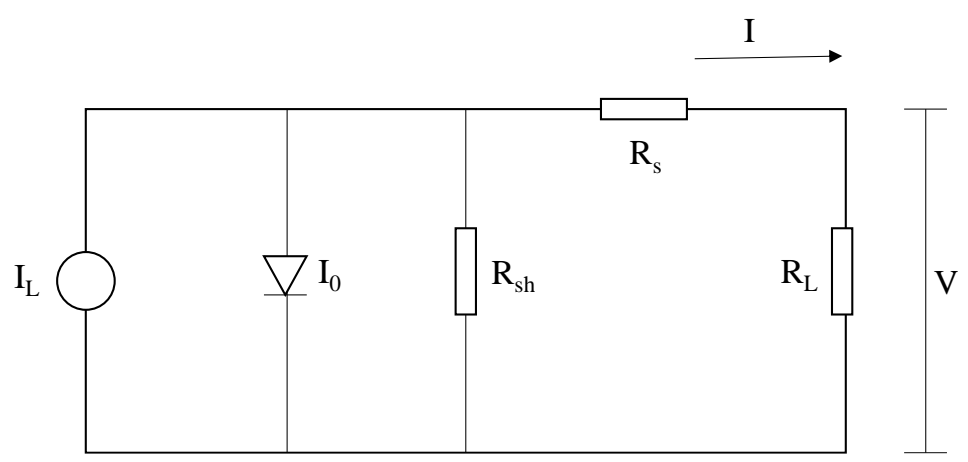

Fig. 1. Schema of one diode equivalent simplified circuit closed on a resistive load $R_{L}$.

Based on this simplified circuit, the mathematical model of a photovoltaic cell can be defined in accordance with the following expression that permits to retrieve the $I-V$ curve:

$$
I=I_{L}-I_{0}\left(e^{\frac{V+I \cdot R_{s}}{n T_{c}}}-1\right)-\frac{V+I \cdot R_{s}}{R_{s h}}
$$

in which $I_{L}$ depends on the solar irradiance, $I_{0}$ is the diode reverse saturation current and is affected by the silicon temperature, $n$ is the ideality factor and $T_{c}$ is the cell temperature $[\mathrm{K}]$.

As it is well known, the performance of a photovoltaic panel is defined according to the "peak power", which identifies the maximum electric power supplied by the panel when it receives an insolation of $1 \mathrm{~kW} / \mathrm{m}^{2}$ at a cell temperature of $25^{\circ} \mathrm{C}$. In actual conditions, it is essential to evaluate the operating conditions under all possible circumstances of irradiance $G$, cell temperature $T_{c}$, wind speed $W$, air temperature $T_{\text {air }}$ and electric load $R_{L}$.

The $T_{c}$ temperature thus is a key parameter that affects the energy conversion efficiency of a PV panel: increasing the temperature, the delivered power decreases. In 
literature, there are several available empirical correlations to obtain the PV panel operating temperature and these correlations have been developed for common geometries and weather conditions. From a mathematical point of view, the correlations for the PV operating temperature are either in explicit or implicit form; in the latter case, an iteration procedure is necessary for the calculation. Most of the correlations typically include the reference conditions and the corresponding values of the pertinent variables [19].

\subsection{Impact of solar irradiance and temperature on the $I-V$ and $P-V$ curves}

For given values of $G, T_{c}$ and $R_{L}$, the operating point can be identified by drawing lines of the different loads $R_{L}$ on the $I-V$ characteristics.

In Figure 2 and Figure 3, it is possible to observe how the intersection between the load line and PV characteristics corresponds to the working point; with the same graphical method, it is possible to identify the working point in terms of electric power. The red circles indicate the locus of maximum power output points.
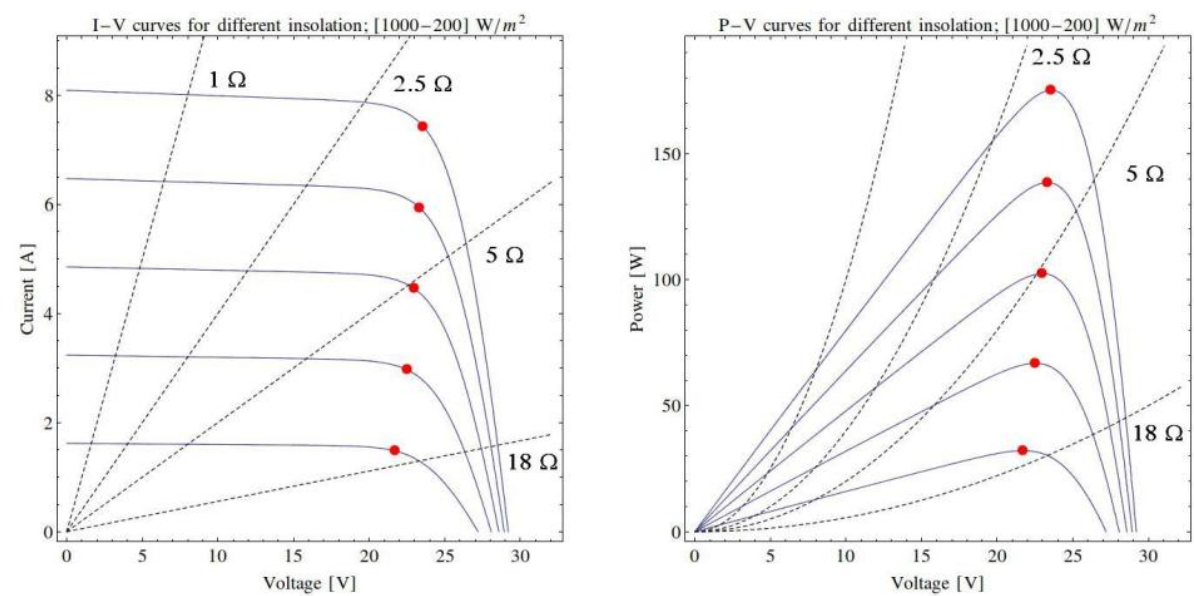

Fig. 2. Working point of a generic PV panel at constant temperature $\left(25^{\circ} \mathrm{C}\right)$ varying insolation and electric load. 

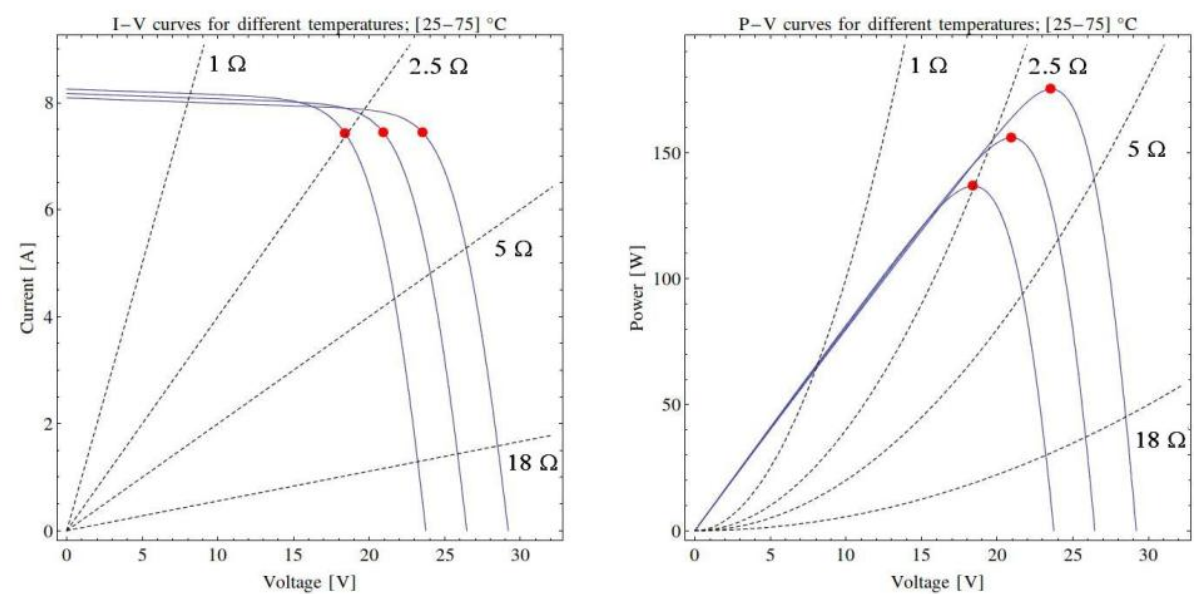

Fig. 3. Working point of a generic PV panel at constant irradiance $\left(1000 \mathrm{~W} / \mathrm{m}^{2}\right)$ varying temperature and electric load.

The solar energy conversion into electrical energy is obviously influenced by the operation point of the panel [20].

\section{$4 \quad$ Artificial Neural Network application}

As shown by the previous considerations, the thermo-electrical behavior of a PV panel is a complex function of the actual climatic conditions and of the cell characteristics.

The complexity of this physical phenomenon does not allow a complete and accurate analytical representation of the thermo-electric balance of the PV panel and the performance assessment generally follows two paths:

1. a simplification of the thermo-electric balance by using empirical correlations;

2. an application of adaptive systems that learn from a large amount of monitored data.

ANNs are distributed, adaptive, generally nonlinear learning machines built from many different processing elements similar to biological neurons. Each artificial neuron (AN) receives connections from other ANs and/or itself. The structure of these connections defines the ANN topology. The signals flowing on the connections are scaled by adjustable parameters called weights and the values of these weights are updated during the training phase. The ANs sum all contributions and produce an output. The outputs of each AN can be either system outputs or inputs sent to the same or other ANs.

In this work, the authors have tested the use of ANNs to predict the operating temperature of a PV panel using the data monitored in a test facility. Different ANNs topologies and typologies were tested. 


\subsection{Data for training and testing}

A large database of specific data that represent the analyzed physical system is required to construct an adaptive system. In order to forecast the temperature of a PV panel, an experimental system (Figure 4) was installed on the roof of the Energy Department of the University of Palermo. The test facility and the monitoring system consist of the following equipment:

- a photovoltaic panel (Kyocera KC175-GH-2),

- a precision resistance set,

- a multimeter Fluke189/FVF2,

- a Delta Ohm pyranometer mod. LP PYRA 02 AV linked to

- an Advantech ADAM 6024 module,

- a Davis Vantage PRO2 Plus Weather station; more details concerning the test facility are explained in [21].

The PV panels and the pyranometer were tilted at an angle that is equal to the latitude of the location ( $38^{\circ}$ South). The electrical load $R_{L}$ was obtained by precision resistances, and the current was calculated on the basis of the measured voltage, accepting the error due to the resistances value. The silicon temperature was measured using thermocouples (type T, copper-constantan) [22] installed at the rear film of the panel [23].

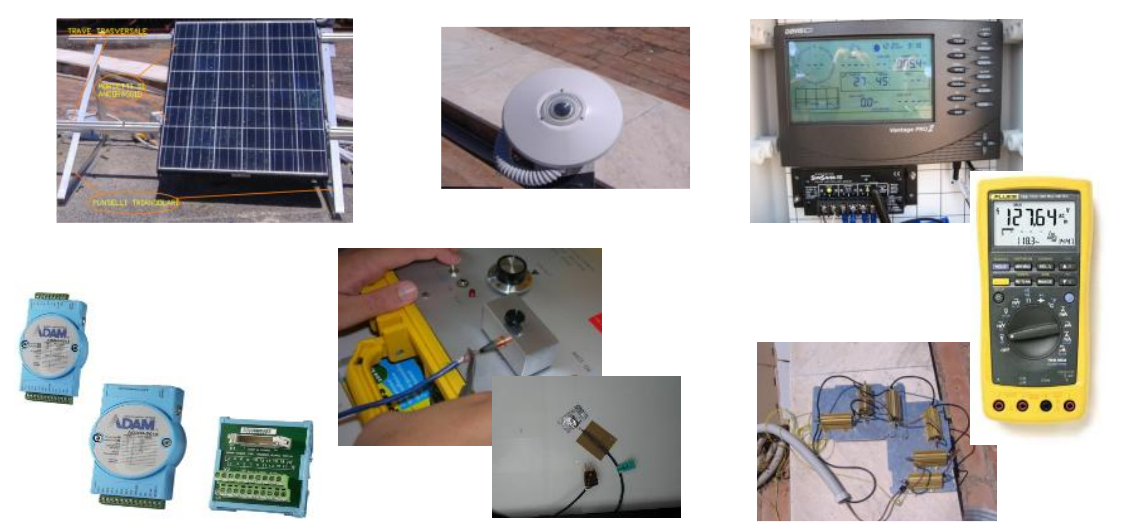

Fig. 4. Experimental system.

All data were collected every 30 minutes and stored for further calculations and comparisons. The physical data used for the training of the ANN were:

- Air temperature $\left[{ }^{\circ} \mathrm{C}\right] ;$

- Solar irradiance $\left[\mathrm{W} / \mathrm{m}^{2}\right]$;

- Wind speed $[\mathrm{m} / \mathrm{s}]$;

- Voltage [V];

- Power output [W];

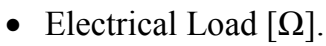


The process by which the knowledge of the physical system is transferred to the adaptive system is carried out in the training phase. During the training phase of an ANN, a set of known input-output vectors are presented to the network updating some mathematical entities. The subsequent testing phase will assess the quality of the neural network model comparing the output with the real data belonging to a dataset not used in the training phase.

\subsection{Preliminary analysis of the collected data}

It is possible to find a wide range of ANNs characterized by different topologies and typologies. Before choosing the neural topology, all data are subject to a preprocessing step that consists in a preliminary analysis that permits to identify possible outliers, to remove unreliable values, to carry out a statistical analysis, and to perform a correlation analysis. After the pre-processing step, the database is validated and the correlation analysis permits a first evaluation of the mutual relationships among the considered variables.

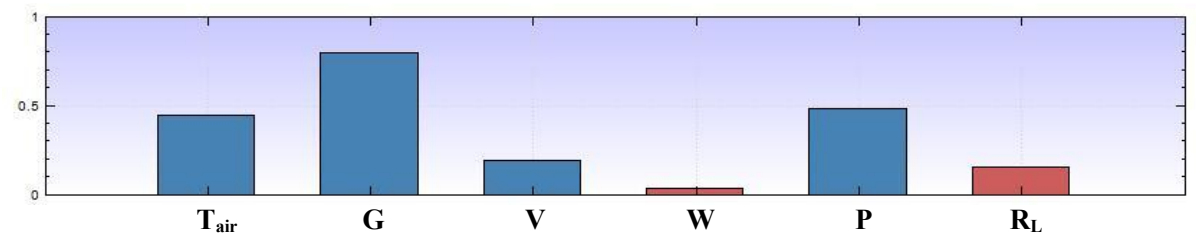

Fig. 5. Correlation analysis between operating temperature and all input data.

Figure 5 shows the linear correlation between $T_{c}$ and all the other features. The higher the bar goes, the more the features are correlated. Blue bars indicate a positive correlation while red bars indicate a negative correlation. The preliminary correlation analysis identified a strong positive correlation between $T_{c}$ and the solar irradiance; on the contrary, a weak negative correlation with the wind speed was detected. Furthermore, a moderate positive correlation with electrical power, air temperature and voltage was found.

A statistical analysis permitted to assess the maximum (Max), mean (Mean) and minimum (Min) values and the standard deviation (StDev) of all considered features (Table 1).

Table 1. Statistical Evaluation.

\begin{tabular}{lccccccc}
\hline & $\begin{array}{c}\mathbf{T}_{\text {air }} \\
{\left[{ }^{\circ} \mathbf{C}\right]}\end{array}$ & $\begin{array}{c}\mathbf{T}_{\text {cell }} \\
{\left[{ }^{\circ} \mathbf{C}\right]}\end{array}$ & $\begin{array}{c}\mathbf{G} \\
{\left[\mathbf{W} / \mathbf{m}^{2}\right]}\end{array}$ & $\begin{array}{c}\mathbf{V} \\
{[\mathbf{V}]}\end{array}$ & $\begin{array}{c}\mathbf{W} \\
{[\mathbf{m} / \mathbf{s}]}\end{array}$ & $\begin{array}{c}\mathbf{P} \\
{[\mathbf{W}]}\end{array}$ & $\begin{array}{c}\mathbf{R}_{\mathbf{L}} \\
{[\mathbf{\Omega}]}\end{array}$ \\
\hline Max & 32.60 & 64.97 & 1221.00 & 28.01 & 8.00 & 167.09 & 18.00 \\
Min & 9.80 & 14.43 & 44.93 & 3.90 & 0.00 & 15.20 & 1.00 \\
Mean & 19.98 & 36.52 & 675.01 & 19.31 & 2.30 & 70.75 & 7.18 \\
StDev & 3.49 & 7.643 & 262.86 & 6.72 & 1.41 & 40.54 & 5.59 \\
sample & 3432 & 3432 & 3432 & 3432 & 3432 & 3432 & 3432 \\
\hline
\end{tabular}


In the following Training Step, the authors decided not to consider the Voltage and Electric load input because their values are already computed in the Electrical power value; the tested ANNs will consider as input only a vector with four components ( $T_{\text {air }}, G, P$ and $W$ ) and as output the $T_{c}$. The training dataset is composed by 2827 vectors and the testing dataset consists in 605 vectors to be used in the validation phase. After the pre-processing phase, it was possible to choose the topology of neural network; different simulations relating to several topologies of ANNs have been tested but in this work only the best ANN solutions will be described: the Gamma two Layer, the Recurrent one Layer and the MLP two Layer. For each topology the design and the algorithm are analyzed, each neural networks was trained and was validated with a post processing phase.

\subsection{Gamma two Layer}

A Gamma ANN is characterized by special memory ANs. The memory AN receives several inputs and produces multiple outputs which are delayed versions of the combined input. This feature has a biological interpretation because, when the biological neuron receives multiple connections, the signal propagation is delayed [24].

As it is possible to see in Figure 6, the proposed Gamma topology is composed of two data sources block (input and output), three gamma memory blocks, three function layer blocks, three weight layer blocks and one delta terminator block. The function layer can be seen as non-linear thresholds for the propagation of the signals. They give the adaptive system its non-linear computing capabilities. The weight layer represents the long-term memory of the system and is adjusted during the learning phase. Finally, the delta terminator is an error criterion block that takes two signals and compares them according to a specific criterion. The appellation "Terminator" means that the signals terminate to flow across the system.

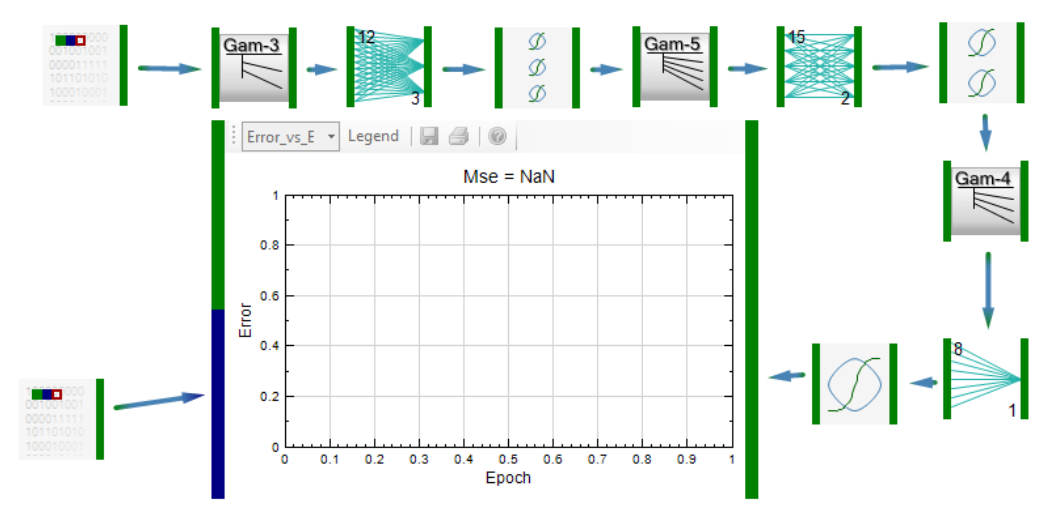

Fig. 6. Gamma two Layer layout. 
After the training, the post-processing phase evaluates the error (Figure 7) and the absolute error (Figure 8) between the measured operating temperature data and the calculated output employing the remaining 605 vectors.

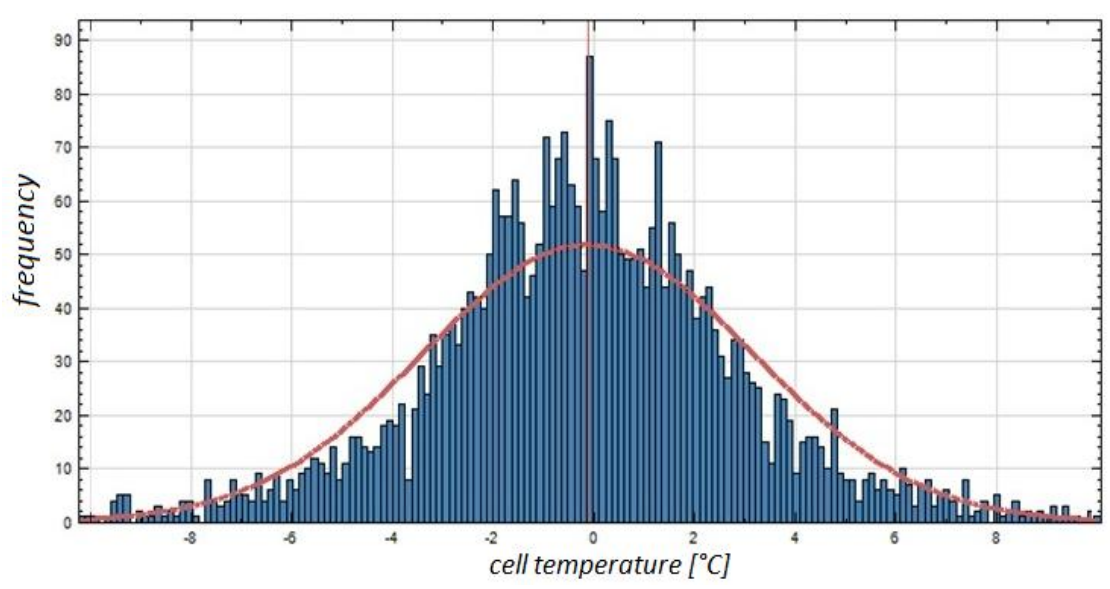

Fig. 7. Error distribution over 605 vectors of $T_{c}$ with Gamma two Layer topology

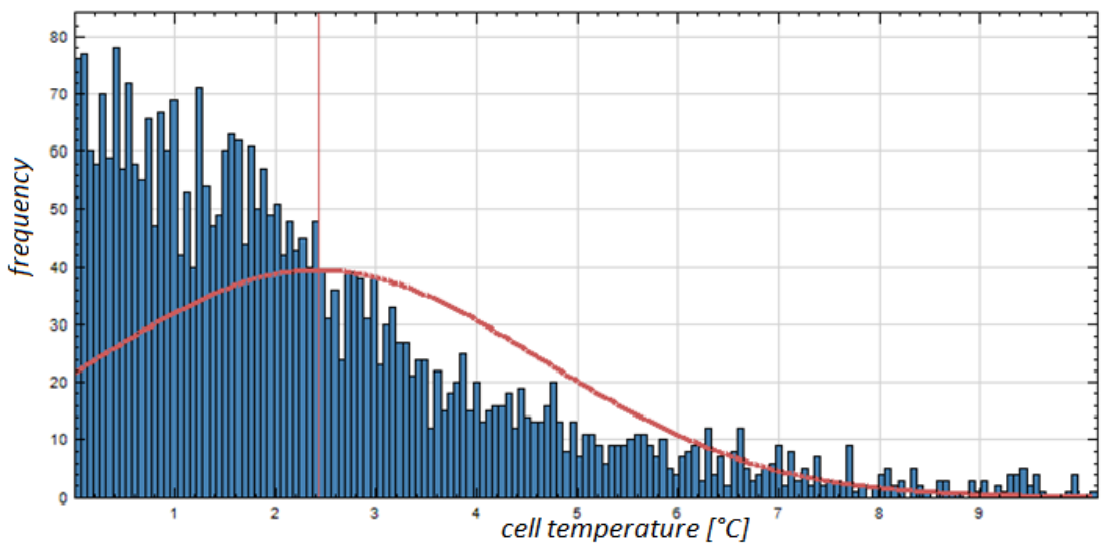

Fig. 8. Absolute Error distribution over 605 vectors of $T_{c}$ with Gamma two Layer topology

The values of the Mean Error (ME) and Mean Absolute Error (MAE) of the Gamma two Layer topology are reported in Table 2.

Table 2. Mean Error and Mean Absolute Error of the Gamma two Layer topology.

\begin{tabular}{ccc}
\hline \multicolumn{3}{c}{ Gamma two Layer } \\
\hline & ME & MAE \\
{$\left[{ }^{\circ} \mathbf{C}\right]$} & -0.119 & 2.428 \\
StDv & 3.286 & 2.217 \\
\hline
\end{tabular}


As shown in Figure 9 a Confidence plot of $\pm 4.408{ }^{\circ} \mathrm{C}$ contains $95 \%$ of the outputs.

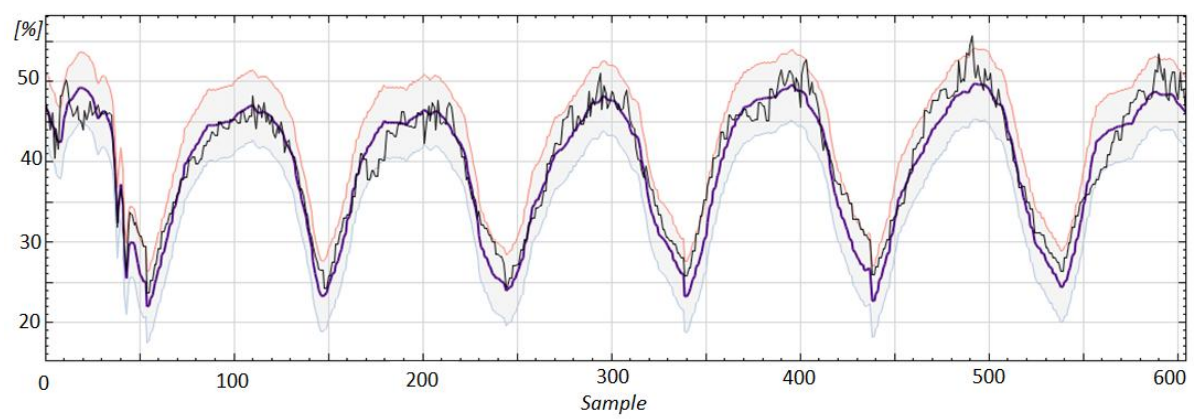

Fig. 9. Confidence Plot of calculated output versus measured data of Gamma two Layer topology

The black line, which represents the calculated output, well follows the trend of the purple line that represents the experimental output. The $95 \%$ confidence is delimited by the red (high) and blue (low) lines.

\subsection{Multi-Layer Perceptron (MLP) two Layer}

A (MLP) is a kind of ANN consisting of multiple layers of ANs in a directed graph, with each layer fully connected to the next one. Except for the input ANs, each node is a neuron with a non-linear activation function. A MLP utilizes a common supervised learning technique called back-propagation for training the network. This topology is one of the simplest available for ANNs. In our work, the MPL two Layer is composed by: two data sources blocks (input and output), two function layer blocks, two weight layer blocks and one delta terminator block as shown in Figure 10.

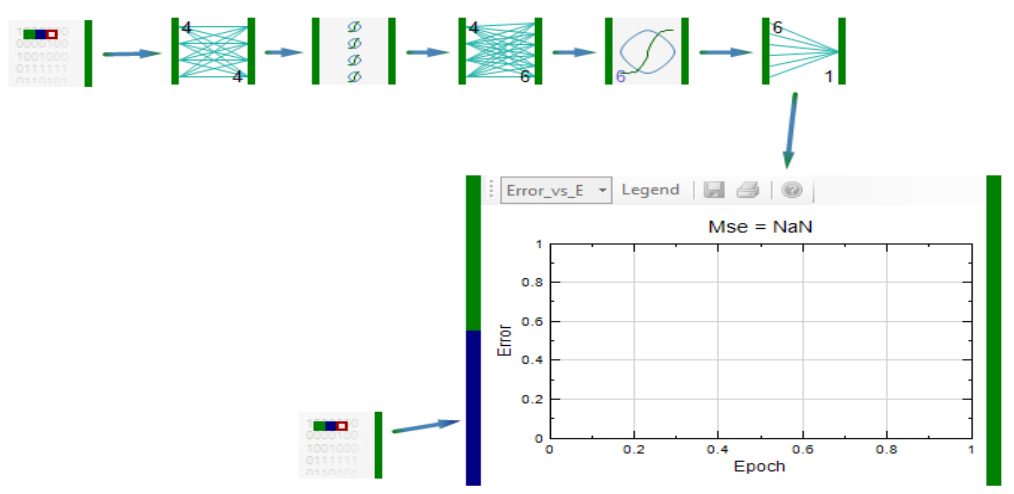

Fig. 10. MLP two Layer topology 
After the training, the post-processing phase evaluates the error and the absolute error between the measured and the calculated operating temperature data. The cumulative results are reported in Table 3.

Table 3. Mean Error and Mean Absolute Error of the MLP two Layer

\begin{tabular}{ccc}
\hline \multicolumn{3}{c}{ MLP two Layer } \\
\hline & ME & MAE \\
{$\left[{ }^{\circ} \mathbf{C}\right]$} & 0.207 & 2.476 \\
StDv & 3.407 & 2.349 \\
\hline
\end{tabular}

For the MLP two Layer topology the confidence plot that contains the $95 \%$ of the outputs is of $\pm 3.936^{\circ} \mathrm{C}$ (Figure 11).

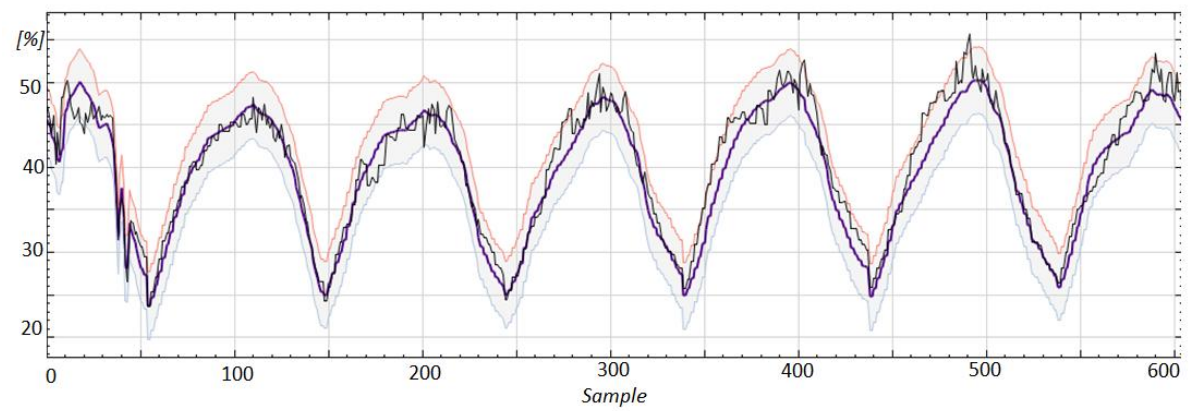

Fig. 11. Confidence Plot of calculated output versus measured data of MLP two Layer topology

\subsection{Recurrent MLP one Layer}

The Recurrent MLP one Layer is a simple ANN topology that employs a recursive flow of the signal to preserve and to use the temporal sequence of events as useful information. This topology (Figure 12) is composed of two data sources blocks (input and output), two weight layer blocks, two function layer blocks and one delta terminator block. 


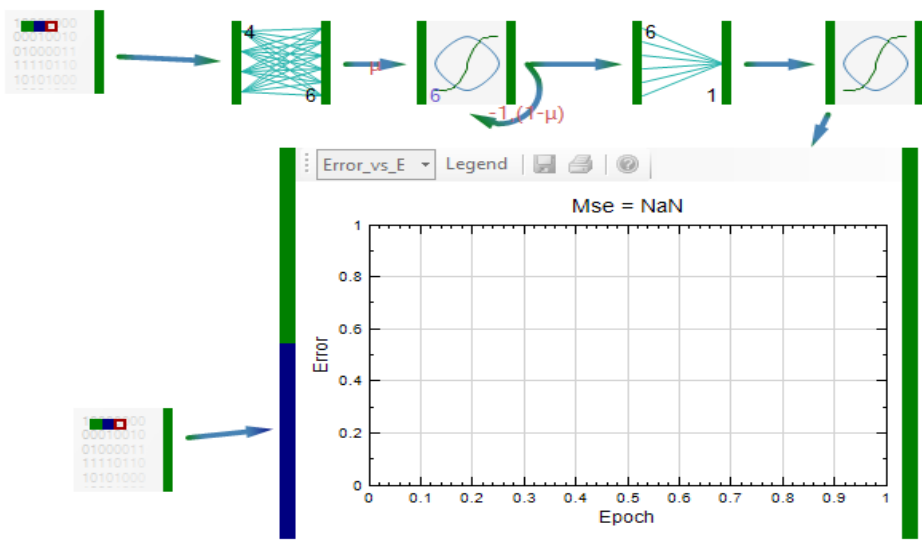

Fig. 12. Recurrent MLP one Layer topology.

Figure 12 iconizes a feedback connection where $\mu$ is the weight of the feedback used to scale the input. Of course, there are different values of $\mu$ for each signal flowing into the first function block layer. After the opportune training phase, the following results are observed:

Table 4. Error Distribution of the Recurrent MLP one Layer topology.

\begin{tabular}{ccc}
\hline & \multicolumn{2}{c}{ Recurrent MLP one Layer } \\
\hline & ME & MAE \\
{$\left[{ }^{\circ} \mathbf{C}\right]$} & 0.229 & 2.489 \\
StDv & 3.436 & 2.386 \\
\hline
\end{tabular}

For the Recurrent MLP one Layer topology the confidence plot that contains the $95 \%$ of the outputs is $\pm 4.517^{\circ} \mathrm{C}$, as shown in Figure 13 .

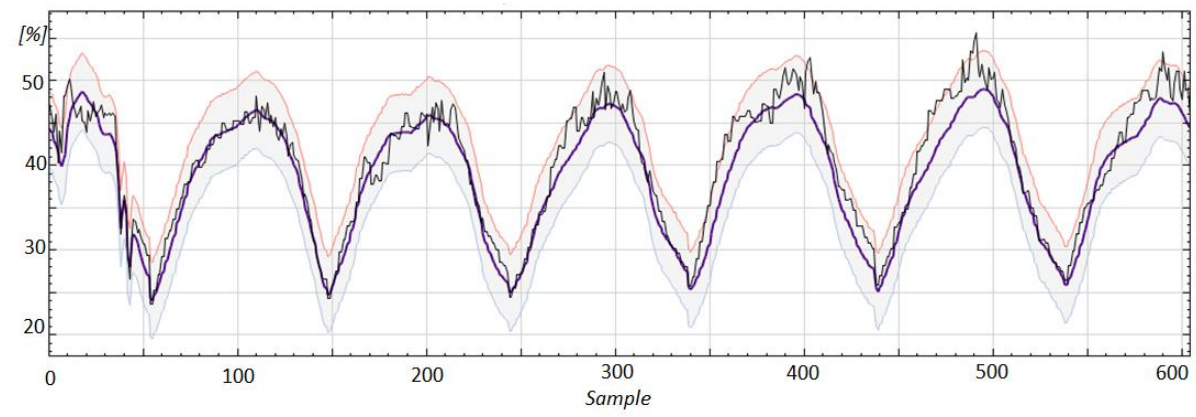

Fig. 13. Confidence Plot of Recurrent one Layer topology 


\section{$5 \quad$ Evaluation of the results}

Each neural network was characterized by the same Input Data (Air Temperature, Wind Speed, Solar Irradiance and Electric Power) and was trained with a dataset of 2827 vectors. The Gamma two Layer and the Recurrent MLP two Layer are two ANN typologies that, with different approaches, have the capability to preserve the temporal sequence of data (memory), while the MLP two Layer is a static ANN. The results show that all the considered ANNs provide a reliable model that is able to fit well with the experimental trends. Generally, the ME is about $\pm 0.2{ }^{\circ} \mathrm{C}$ and the MAE is close to $2.4^{\circ} \mathrm{C}$. The training phase of Gamma two Layer ANN requested 1 minute, while the other two typologies requested a shorter time.

In literature [19, 25-29], there are different models that allow calculating the operating temperature. In order to validate the neural network approach, the authors made a comparison between the values of $T_{c}$ obtained by the three previously illustrated networks and the operating temperature calculated by one of the most cited empirical correlation:

$$
T_{c}=T_{a}+\alpha G \cdot\left(1+\beta T_{a}\right) \cdot(1-\gamma W) \cdot\left(1-1.053 \eta_{c}\right)
$$

where $\eta_{c}$ is the efficiency of a PV panel and $\alpha, \beta$ and $\gamma$ are three constants ( $\alpha=38.0385, \beta=3.15126, \gamma=2.64173$ ) [24].

The comparison, as represented in Table 5, shown that the empirical correlation achieves a MAE that is about twice compared to the ANN results.

Table 5. Comparison between the ANN and empirical correlation results.

\begin{tabular}{ccc}
\hline ANN & $\begin{array}{c}\text { Training } \\
\text { time }\end{array}$ & MAE \\
\hline Gamma two Layer & $\approx 1 \mathrm{~min}$ & 2.428 \\
Recurrent MLP one & $\approx 10 \mathrm{~s}$ & 2.489 \\
Layer & & \\
MLP two Layer & $\approx 20 \mathrm{~s}$ & 2.476 \\
Empirical & - & $\mathbf{4 . 7 1 9}$ \\
\hline
\end{tabular}

\section{Conclusions}

In this paper, an artificial neural network approach has been proposed to determine the operative temperature of a PV panel.

The application of the artificial neural network model represents a simple and fast solution to correctly evaluate the operative regimen of a PV system. To this purpose, different network architectures have been tested and trained with experimental data consisting in: air temperature, wind speed, solar irradiance, power output and cell temperature. The three best solutions of ANNs are reported: Gamma two Layer, MLP two Layer and Recurrent MPL one Layer. 
The results obtained by the ANNs demonstrates that this approach can be considered a reliable tool to forecast the cell temperature of the PV panel. Comparing the performances of these networks with a very often cited empirical model, used for determining the operating temperature of the panel, the ANN approach presents a significant lower MAE. Furthermore, the very short time required by MLP two Layer and Recurrent MPL one Layer for the training phase, suggests that ANNs could be integrated in a software for run-time evaluation.

\section{References}

1. IEA: International Energy Agency; "World Energy Outlook 2012” ISBN: 978-92-6418084-0.

2. Pernick, R., Wilder, C., Clean Tech Nation: How the US Can Lead in the New Global Economy. Harper Business, (2012).

3. Cellura, M., Campanella, L., Ciulla, G., Guarino, F., Lo Brano, V., Cesarini, D. N., Orioli, A., A net zero energy building in Italy: design studies to reach the net zero energy target. In Building Simulation, an IBPSA-AIRAH conference, Sydney, Australia (2011).

4. Beccali, G., Cellura, M., Lo Brano, V., Orioli, A., Single thermal zone balance solved by Transfer Function Method, Energy and buildings, 37(12), 1268-1277, (2005).

5. Ciulla, G., Franzitta, V., Lo Brano, V., Viola, A., Trapanese, M.. Mini Wind Plant to Power Telecommunication Systems: A Case Study in Sicily. Advanced Materials Research, 622, 1078-1083, (2013).

6. Di Dio, V., Favuzza, S., La Cascia, D., Miceli, R.. Economical Incentives and Systems of Certification for the Production of Electrical Energy from Renewable Energy Resources. In Clean Electrical Power, 2007. ICCEP'07, pp. 277-282, IEEE (2007).

7. Cellura, M., Ciulla, G., Lo Brano, V., Marvuglia, A., Orioli, A., A Photovoltaic panel coupled with a phase changing material heat storage system in hot climates. PLEA 2008, $25^{\text {th }}$ Conference on Passive and Low Energy Architecture, Dublin (2008).

8. Tiwari, A., Sodha, M.S., Performance evaluation of hybrid PV/thermal water/air heating system: A parametric study. Renewable Energy 31, pp. 2460-2474, (2006).

9. Zakharchenko, R., Licea-Jimenez L., Perez-Garc S.A., Vorobiev, P., Dehesa-Carrasco, U., Perez-Robles, J.F., Gonzalez-Hern J., Vorobiev Yu., Photovoltaic solar panel for a hybrid PV/thermal system. Solar Energy Materials \& Solar Cells 82, pp. 253-261, (2004).

10. Eicker, U., Solar Energy for Buildings.1 th edition Wiley. ISBN:0-471-48637-X.

11. Cellura, M., Lo Brano, V., Mistretta, M., Orioli, A. To Assess the Validity of the Transfer Function Method: A Neural Model for the Optimal Choice of Conduction Transfer Functions. ASHRAE Transactions, 116(2), 585. (2010).

12. Ciulla, G., Lo Brano, V., Orioli, A., A criterion for the assessment of the reliability of ASHRAE conduction transfer function coefficients, Energy and Buildings, vol. 42, Issue 9, pp. 1426-1436, (2010).

13. Haykin, S., Neural Networks: A Comprehensive Foundation" $2^{\text {nd }}$ Edition. Prentice Hall, 1999.

14. Bishop C., Pattern Recognition and Machine Learning, Springer, 2006.

15. Yang, Y., Elfayoumy, S., Anti-Spam Filtering Using Neural Networks and Baysian Classifiers. Proceedings of the 2007 IEEE International Symposium on Computational Intelligence in Robotics and Automation. Jacksonville, FL, USA (2007).

16. Rowley, H.A., Baluja, S., Kanade, T., Neural Network-Based Face Detection. IEEE Transactions on Pattern Analysis and Machine Intelligence, 20, pp.23-38 (1998). 
17. Venayagamoorthy, G.K., Moonasar, V., Sandrasegaran, K., Voice recognition using neural networks. Proceedings of the 1998 South African Symposium on Communications and Signal Processing, Cape Town (1998).

18. Hui-Feng Tsai, Huan-Liang Tsai, Implementation and verification of integrated thermal and electrical models for commercial PV modules. Solar Energy; vol. 86, pp.654-665 (2012).

19. Skoplaki, E., Palyvos, J.A., On the temperature dependence of photovoltaic module electrical performance: A review of efficiency/power correlations. Solar Energy, vol.83, pp. 614-624 (2009).

20. Lo Brano, V., Ciulla, G., Franzitta, V., Viola, A. A novel implicit correlation for the operative temperature of a PV panel. 2012 AASRI Conference on Power and Energy Systems (2012).

21. Lo Brano, V., Orioli, A., Ciulla, G., Culotta, S., Quality of wind speed fitting distributions for the urban area of Palermo, Italy, Renewable Energy, vol. 36, pp. 1026-1039 (2011).

22. Cardona, E., Piacentino A., A measurement methodology for monitoring a CHCP pilot plant for an office building. Energy and Buildings, vol.35 (9), pp.919-925 (2003).

23. Lo Brano, V., Orioli, A., Ciulla, G., On the experimental validation of an improved fiveparameter model for silicon photovoltaic modules, Solar Energy Materials and Solar Cells, vol. 105, pp. 27-39 (2012).

24. Principe, J.C., Euliano N.R., Lefebvre W.C., Neural and Adaptive Systems, Fundamentals though Simulations, John Wiley \& Sons, Inc. ISBN 0-471-35167-9.

25. Skoplaki, E., Palyvos, J.A., Operating temperature of photovoltaic modules: A survey of pertinent correlations. Renewable Energy, vol.34 (1), pp. 23-29 (2009)

26. Servant, JM., Calculation of the cell temperature for photovoltaic modules from climatic data. $9^{\text {th }}$ biennal congress of ISES-Intersol 85, Montreal, Canada, 370 (1985).

27. Duffie, J.A., Beckman, EA., Solar energy thermal processes. 3nd ed. Hoboken (NJ):Wiley (1991).

28. Hove, T., A method for predicting long-term average performance of photovoltaic systems. Renewable Energy, vol. 21, pp.207-29 (2000).

29. Tiwai, GN., Solar Energy-Fundamentals, design, modeling and applications. Pang Bourne (UK): Alpha Science, 450, (2002). 